the CLAP (clathrin and AP-2 binding) domain and functions at the level of synaptic vesicle endocytosis. Here, we assessed the impact of BIN1 neuronal isoform on APP metabolism. Because it has been reported that BIN1 could interact with CLIP-170, a microtubule-associated protein, we also assessed the ability of BIN1 to interact with tau. Methods: Analysis of APP metabolism SKNSH-SY5Y-APP695 and HEK293-APP695 were transfected for 48 hours. Holo-APP and APP-CTF were measured by protein blotting and secreted metabolites were measured by ELISA.Costaining and Co-immunoprecipitation. We assessed the potential colocalization between BIN1 and APP or tau by confocal images from immunofluorence staining in SKNSH-SY5Y cells. Using co-immunoprecipitation, we investigated whether tau might interact with ubiquitous and/or neuronal BIN1 isoforms. Results: Overexpression of neuronal BIN1 isoform in SKNSHSY5Y or HEK293 cell lines, both stably expressing the APP695wt isoform, did not affect APP metabolism. In addition, neuronal BIN1 isoform did not show any costaining with APP. However, co-immunoprecipitation experiments using HEK293 or SKNSH-SY5Y cells transiently overexpressing BIN1 and tau (1N4R Tau) suggested that anti-tau antibody preferentially co-precipitated the neuronal BIN1 isoform and not an ubiquitous BIN1 isoform. In line with these results, we observed a strong costaining between 1N4R tau and neuronal BIN1 isoform whereas no similar colocalization was found between the 1N4R tau and ubiquitous BIN1 isoform. Moreover, from differentiated neuroblastoma cells endogenously expressing BIN1 and tau, we observed strong labeling of BIN1 and tau in neurites, consistent with a previous report showing that BIN1 is expressed in axonal segments. Conclusions: Here, we reported BIN1 as a new partner of tau. Moreover, based on our observations, tau preferentially interacts with neuronal BIN1 isoform suggested a neuron-specific interaction. Further experiments should be addressed to determine the impact of this interaction on tau pathology.

\section{P3-013 PATTERN OF TAU HYPERPHOSPHORYLATION IN BRAINSTEM NUCLEI OF TAU TRANSGENIC MICE}

Kerstin Morcinek $^{1}$, Kirsten Pilz ${ }^{1}$, Christoph Köhler ${ }^{1}$, Andrea Wevers ${ }^{1}$, Hannsjörg Schröder ${ }^{1}$, Jürgen Götz ${ }^{2}{ }^{1}$ University of Cologne, Köln, Germany; ${ }^{2}$ University of Sydney, Camperdown, Australia.

Background: Recent reports present evidence for the early appearance of brainstem tau pathology in dementia disorders, such as Alzheimer's disease (AD). Our own preliminary studies revealed neurofibrillary lesions in selected brainstem areas in P301L tau-transgenic pR5 mice. To address the question whether this mouse model mirrors the brainstem changes that are observed in dementia disorders, we investigated the degree and distribution of the tau pathology throughout the brainstem. Methods: Coronal brainstem sections of 20-month-old P301L tau transgenic pR5 mice and non-transgenic littermates were screened immunohistochemically for human tau (HT7), the physiologically phosphorylated epitopes Thr231/Ser235 (mAb AT180) and Ser202/Thr205 (mAb AT8), the abnormally phosphorylated epitope Ser422 (mAb pS422) and Gallyas silver-impregnated structures. Results: The expression of the human tau transgene was observed in multiple brainstem nuclei of pR5 mice, particularly in the magnocellular reticular formation, the vestibular nuclei, the cranial nerve motor nuclei, the sensory trigeminal nerve nuclei, the inferior and superior colliculi, the periaqueductal and pontine gray matter, and the red nucleus. Most of the HT7immunoreactive (ir) nuclei also showed hyperphosphorylation of tau at the epitopes Thr231/Ser235 indicating an early stage of neurofibrillary tangle formation. In comparison to HT7 and AT180, fewer AT8-ir neurons as well as the lack of pS422-positive or silver-stained cell bodies suggests the absence of insoluble filamentous deposits, neuropil threads or ghost tangles. Neither the human tau transgene nor hyperphosphorylated tau protein was detected in brainstem nuclei of non-transgenic littermates. Conclusions: Although numerous brainstem nuclei in P301L tau transgenic pR5 mice express human tau protein, the development of neurofibrillary lesions seems to be rare, whereas human tissue shows severe affection of brainstem nuclei in early stages of AD. The pattern of tau pathology throughout the brainstem of $\mathrm{pR} 5$ mice differs largely from $\mathrm{AD}$, while the distribution of affected nuclei partly resembles those seen in other tauopathies, particularly in progressive supranuclear palsy (PSP). Therefore the brainstem of pR5 mice might be a suitable system to examine pathogenic mechanisms in PSP.

\section{P3-015 TAU-DEPENDENT IMMEDIATE EARLY GENES}

Amadeus Gladbach, Yazi Ke, Arne Ittner, Lars Ittner, The University of Sydney, Camperdown, Australia.

Background: Alzheimer's disease (AD) is the most common neurodegenerative disorder, characterized by a progressive loss of cognition. The second most prevalent form, before the age of 65 years, is frontotemporal dementia (FTD). Both AD and FTD are characterized by deposition of hyperphosphorylated forms of the microtubule-associated protein tau forming intracellular aggregates, such as neurofibrillary tangles (NFTs). We and others have recently shown that amyloid- $\beta$ toxicity is tau-dependent in $\mathrm{AD}$ mouse models, and can accordingly be prevented by reducing tau levels or expressing dominant-negative mutant tau (Roberson, Science, 2007; Ittner, Cell, 2010). Mechanistically, we could show that tau couples excitotoxic down-stream signaling to glutamate receptors. Excitotoxic cell damage results in activation of target genes, referred to as immediate early genes (IEG). Methods: Here, we administered pentylenetetrazol (PTZ) to wild-type and tau-deficient (tau -/-) mice to establish which, out of a panel of 32 known excitotoxic IEGs are tau-dependent. The IEG response was determined by quantitative PCR of mRNA obtained from hippocampi of six mice per genotype and time point for up to 24 hours post-injection. Results: Profiling the mRNA levels upon PTZ administration revealed three categories of IEGs; those which show no activation in the absence of tau, those with delayed activation in tau $-/-$, and those activated independent of tau. Conclusions: Taken together, we established a complex pattern of differential tau-dependent gene regulation in response to excitotoxic brain damage, with possible implications for pathological processes in AD and FTD.

\section{P3-016 ER STRESS IN ALZHEIMER'S DISEASE AND PSP: COMMON DISEASE MECHANISM?}

Lauren Stutzbach ${ }^{1}$, Michele Hawk ${ }^{2}$, Sid Gilman ${ }^{3}$, Virginia Lee ${ }^{2}$, John Trojanowski ${ }^{2}$, Gerard Schellenberg ${ }^{2},{ }^{1}$ University of Pennsylvania Perelman School of Medicine, Department of Pathology and Laboratory Medicine, Philadelphia, Pennsylvania, United States, ${ }^{2}$ University of Pennsylvania, Philadelphia, Pennsylvania, United States; ${ }^{3}$ University of Michigan, Ann Arbor, Michigan, United States.

Background: The unfolded protein response (UPR), an indicator and modulator of ER stress, is triggered by the presence of mis- or unfolded proteins in the ER lumen and is comprised of three stress sensors: Ire1, ATF6, and PERK. The PERK arm of the UPR has both adaptive and apoptotic effects, depending on the duration of ER stress. The UPR is activated in several neurodegenerative tauopathies, including Alzheimer's disease (AD), Multiple System Atrophy and Progressive Supranuclear Palsy (PSP). A recent genome-wide association study for PSP indicated that the gene encoding the PERK protein is a risk factor for PSP. Our goal for this study was to compare PERK activation in a panel of brain areas sampled from AD and PSP patients, as well as normal controls to determine the relative strength and localization of this stress response across tauopathies. Methods: We assayed post-mortem brain tissue from AD patients, PSP patients and normal controls for activation of PERK and its downstream effector eIF2 $\alpha$. Using immunohistochemistry, we stained a panel of six brain areas for activated PERK (pPERK) and activated eIF $2 \alpha$ (peIF2 $\alpha$ ): hippocampus, mid-frontal cortex, midbrain, pons, medulla and folia of the cerebellum. We also used double immunofluorescence to assay the hippocampus and the pons for PPERK and hyperphosphorylated tau (htau). Results: Brains affected by AD had the strongest PERK activation in the hippocampus, followed by the mid-frontal cortex and then the three brainstem regions. Conversely, PSP-affected brains had the strongest PERK activation in the pons and the medulla, followed by the midbrain and the hippocampus. PERK was also activated in the hippocampus of clinically normal controls, and the degree of this activation correlated with advancing age and tau burden. pPERK tended to colocalize with 
diffuse, pre-fibrillar htau but not with full-blown tangles. Conclusions: The PERK arm of the UPR is activated most strongly in disease-affected brain regions in AD and PSP. PERK activation in these regions may temporally precede tangle formation in tauopathies.
PPERK activation in the AD hippocampus

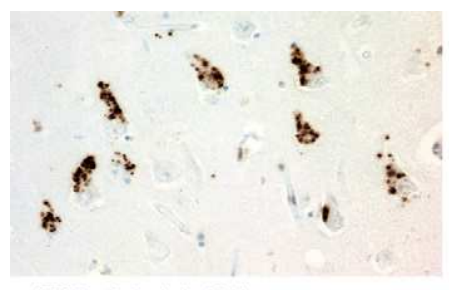

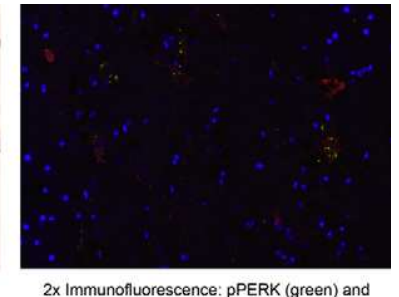

$2 \times$ Immunofluorescence: pPERK
hyperphosphorylated tau (red)

\section{P3-017 OLFACTORY DEFICITS INDUCE} NEUROFILAMENT HYPERPHOSPHORYLATION

Ling-Qiang Zhu, Juan Hu, Dan Liu, Tongji Medical College, Huazhong University of Science and Technology, Wuhan, China.

Background: Olfactory dysfunction, including structural abnormalities of the olfactory epithelium, the olfactory bulb and the central olfactory cortices is recognized as an early feature of Alzheimer disease (AD), the most prevalent neurodegenerative disease in aged population characterized by intracellular neurofibrillary tangles (NFTs). How olfactory deficits are linked with AD-like neuropathological changes is still unknown. Methods: Bilateral olfactory bulbectomy (OBX) rats and Cnga2 -/Y mice were used for the dysfunction of olfactory system and Western blot, immunohistochemistry were performed for analysis the NF phosphorylation. Results: Here, by using two anosmia animal models, bilateral olfactory bulbectomy (OBX) rats and Cnga2 -/Y mice, which lack intact olfactory CNG channels, we found the immunoreactivity of phosphorylated neurofilament (NF) are highly increased in the neurites at both the hippocampus and the cortex. Conclusions: As hyperphosphorylated NF is one of the main components of NFTs, our study strongly suggested the underlying correlation of olfactory deficits with $\mathrm{AD}$-like pathological impairments.

\section{P3-019 REGULATION OF MEMBRANE PROTEIN, LIPID PROFILE AND FLUIDITY BY ZINC DURING ALUMINIUM INDUCED NEURODEGENERATION}

Devinder Dhawan, Neha Singla, Panjab University, Chandigarh, India.

Background: The alterations in the neurometabolism of metals have been regarded as one of the key links on the onset and progression of neurodegenerative diseases. Aluminum is environmentally abundant and a non essential element, which has been implicated in human neurodegenerative diseases including Alzheimer's. On the other hand, Zinc is an important dietary element, which is considered as a vital element for the various physiological and biochemical functions of the central nervous system. The objective of the present study was to investigate the effects of Zinc ( $\mathrm{Zn}$ ) supplementation on lipid profile, proteins and fluidity of cerebrum and cerebellum membranes in aluminium (Al) treated rats. Methods: The study was designed into four different treatment groups. Group I normal control, group II aluminium ( $\mathrm{AlCl} 3$ ) given orally (100 $\mathrm{mg} / \mathrm{kg}$ b.wt./day in drinking water), group III zinc $(\mathrm{ZnSO} 4)$ in drinking water $(227 \mathrm{mg} / \mathrm{L}$ everyday in drinking water) and group IV was given a combined treatment of aluminium and zinc. Results: A significant decrease in the levels of total lipids, glycolipids, phospholipids, cholesterol and gangliosides contents were observed in both the cerebrum and cerebellum following $\mathrm{Al}$ exposure, which however were significantly increased during $\mathrm{Zn}$ supplementation. Moreover, $\mathrm{Al}$ treatment increased the conjugated diene formation which was observed to be reduced upon $\mathrm{Zn}$ supplementation. Further, Al treatment significantly elevated the fluorescence polarization, anisotropy and order parameter, which were normalized following $\mathrm{Zn}$ administration to $\mathrm{Al}$ treated animals. Moreover, $\mathrm{Al}$ treatment increased the protein expressions of glial fibrillary acid protein (GFAP), amyloid precursor-protein (APP) and tau protein, which were found to be reversed upon $\mathrm{Zn}$ administration in both the regions of brain. Conclusions: The present study depicts the potential of $\mathrm{Zn}$ in containing the adverse effects caused by $\mathrm{Al}$ on membrane composition (lipids and proteins) and fluidity in rat brain.

\section{P3-020 REGULATION OF TAU EXPRESSION BY MIR-132}

Pascal Smith, Sebastien Hebert, Centre de Recherche du CRCHUQ-CHUL, Université Laval, Quebec City, Quebec, Canada.

Background: It is well established that changes in the regulation of tau expression and/or metabolism are involved in the development of Alzheimer's disease and related tauopathies. Despite intensive investigation, however, little is know about the molecular mechanisms that participate in the transcriptional and post-transcriptional regulation of endogenous tau, especially in neurons. We have recently shown that microRNAs, and in particular miR-132, play an important role in the regulation of tau exon 10 alternative splicing by modulating neuronal polypyrimidine-tract binding protein 2 (PTBP2). Interestingly, miR-132 is also predicted to target the 3' untranslated region (3'UTR) of tau, suggesting that tau expression is directly regulated by microRNAs. Methods: We used mouse neuroblastoma 2A (N 2 A) cells as biological model. These cells were transfected with either miR-132 mimics or inhibitors. Endogenous tau expression was assessed by Western blot and quantitative RT-PCR. These experiments were complemented with luciferase reporter assays. Results: As hypothesized, overexpression of miR-132 decreased neuronal tau protein and mRNA levels, while the opposite effects were observed in response to the inhibition of miR-132. We could confirm the direct interaction between miR-132 and the murine tau 3'UTR. Interestingly, PTBP1 and 2 directly affected tau at the transcriptional level. Conclusions: Our results strongly suggest that miR-132 modulates tau metabolism through direct and indirect pathways. Further experiments are underway to understand the network between miR-132 and its targets, including tau.

\section{P3-021 DEXMEDETOMIDINE INDUCES TAU HYPERPHOSPHORYLATION IN THE MOUSE HIPPOCAMPUS}

Robert Whittington ${ }^{1}$, László Virág ${ }^{1}$, Carl Julien ${ }^{2}$, Noura B. El Khoury ${ }^{2}$, François Marcouiller ${ }^{2}$, Françoise Morin ${ }^{2}$, Emmanuel Planel ${ }^{2},{ }^{1}$ Columbia University, New York, New York, United States; ${ }^{2}$ Université Laval, CHUL, Québec City, Quebec, Canada.

Background: In Alzheimer's disease and other neurodegenerative tauopathies, the microtubule-associated protein tau can undergo aberrant phosphorylation, potentially leading to its aggregation and the development of neurofibrillary pathology. Anesthetics can induce tau hyperphosphorylation through a mechanism involving the inhibition of protein phosphatase $2 \mathrm{~A}$ activity by anesthesia-induced hypothermia. However, we recently demonstrated that the commonly used anesthetic propofol induces tau hyperphosphorylation in the absence of hypothermia. The aim of this study was to determine the impact of normothermic administration of dexmedetomidine, a commonly used sedative-analgesic agent, on tau phosphorylation in vivo. Methods: Following institutional approval, male C57BL6/J mice (8-10 week old) received either dexmedetomidine (Dex) $300 \hat{\mathrm{I}} 1 / 4 \mathrm{~g} / \mathrm{kg}$ or $0.9 \%$ saline (control) i.p. The Dex-treated mice were sacrificed $30 \min (n=6)$ and $2 \mathrm{~h}(\mathrm{n}=6)$ following treatment and control $(n=6)$ mice were sacrificed at $30 \mathrm{~min}(0.5 \mathrm{~h})$. Hippocampal tissue was immediately harvested at the end of the study, and levels tau phosphorylated (p-tau) at the AT8 (pSer 202/pThr 205), CP13 (pSer 202), and PHF-1 (pSer 396/pSer 404) phosphoepitopes, as well as total tau were determined using SDS-PAGE followed by immunoblotting. The mechanism of tau hyperphosphorylation was dissected by screening for the activation pattern of an extensive panel of tau kinases and phosphatases. Statistical 